\title{
Newly developed adoptive cell therapy using autologous tumor-infiltrating lymphocytes in cervical cancer
}

\author{
Takashi Iwata, Guanliang Chen, Hiroshi Nishio, Yuya Nogami, Yuki Kato, Yo Sugawara, Ikumo Tanaka, \\ Tomoya Matsui, Masaki Sugawara, Daisuke Aoki
}

Keio University School of Medicine, Tokyo, Japan (iwatatakashi@1995.jukuin.keio.ac.jp)

Objective: Adoptive cell therapy using tumor-infiltrating lymphocytes (TIL-ACT) is a promising immunotherapy using autologous lymphocytes ex vivo expanded from patient's tumor. We are performing a phase I study for malignant melanoma and then perform a phase II study for recurrent cervical cancer as to evaluate the safety and efficacy of TIL-ACT.

Methods: Three patients with malignant melanoma received TIL-ACT. Tumor fragments were cultured in outgrowth medium to produce TIL. Then T cell populations with tumor reactivity were selected for rapid expansion, generating over 1,000-fold TILs within 2 weeks and finally reinfused into the patient who received preparative lymphodepleting. IL-2 regimens after cell transfer were conducted to promote TIL growth and antitumor activity. The primary endpoint was to define treatment feasibility as completion of TIL-ACT without early cessation due to unacceptable adverse events. The secondary endpoints were safety assessed using CTCAE v. 4.0, clinical response; objective response rate based on the RECIST v.1.1.

Results: Three cases of TIL-ACT treated melanoma patients were successfully completed without unacceptable adverse events including one partial response, one stable disease and one progression disease case. Next, we manufactured a TIL products from specimens of three cervical cancer patient, and succeeded in producing TIL that meets the standards of TIL-ACT. Based on this result, TIL-ACT for cervical cancer was approved by the Japanese Ministry of Health, Labour and Welfare as advanced medical treatment. Conclusion: TIL-ACT could be safely performed for Japanese patients with malignant melanoma. Currently, we are implementing TIL-ACT for recurrent cervical cancer. 